\title{
Optical Nature of Non-Substituted Triphenylmethyl Cation: Crystalline State Emission, Thermochromism, and Phosphorescence
}

\author{
Tomohiko Nishiuchi, ${ }^{*[a, b]}$ Hikaru Sotome,${ }^{[c]}$ Risa Fukuuchi, ${ }^{[d, e]}$ Kenji Kamada,,${ }^{[d, e]}$ Hiroshi Miyasaka, ${ }^{[c]}$ and \\ Takashi Kubo *[a,b] \\ [a] Dr. T. Nishiuchi, Prof. Dr. T. Kubo \\ Department of Chemistry, Graduate School of Science, Osaka University, 1-1 Machikaneyama, Toyonaka, Osaka, 560-0043, Japan \\ E-mail: nishiuchit13@chem.sci.osaka-u.ac.jp \\ [b] Dr. T. Nishiuchi, Prof. Dr. T. Kubo \\ Innovative Catalysis Science Division, Institute for Open and Transdisciplinary Research Initiatives, (ICS-OTRI), Osaka University, Suita, Osaka, 565-0871, \\ Japan \\ [c] Dr. H. Sotome, Prof. Dr. H. Miyasaka \\ Division of Frontier Materials Science and Center for Advanced Interdisciplinary Research, Graduate School of Engineering Science, Osaka University, \\ Toyonaka, Osaka, 560-8531, Japan \\ [d] Ms. R. Fukuuchi, Prof. Dr. K. Kamada \\ Nanomaterial Research Institute (NMRI), National Institute of Advanced Industrial Science and Technology (AIST), Ikeda, Osaka 563-8577, Japan \\ [e] Ms. R. Fukuuchi, Prof. Dr. K. Kamada \\ Department of Chemistry, Graduate School of Science and Technology, Kwansei Gakuin University, Sanda, Hyogo, 669-1337, Japan
}

\begin{abstract}
Since the discovery of the triphenylmethyl (trityl) cation 120 years ago, a variety of aromatic cations having various colors and luminescence properties have been rigorously studied. Many, differently substituted trityl cations have been synthesized and their optical properties have been elucidated. However, the optical properties of the parent, non-substituted and highly reactive trity cation, which was observed to be very weakly luminescent, have not been subjected to detailed investigation. In the effort described herein, we explored the optical nature of non-substituted trityl hexafluorophosphate $\left(\mathrm{PF}_{6}\right)$ in the crystalline state. Trityl $\mathrm{PF}_{6}$ was found to exist as two crystal polymorphs including a yellow $(\mathbf{Y})$ and an orange $(\mathbf{O})$ form. Moreover, we observed that these crystalline forms display crystalline-state emission with different colors. The results of X-ray crystallographic analysis showed that the two polymorphs have totally different molecular packing arrangements. Furthermore, an investigation of their optical properties revealed that the O-crystal undergoes a distinct color change to yellow upon cooling as a consequence of a change in the nature of the charge transfer interaction between the cation and $\mathrm{PF}_{6}$ anion, and that both the $\mathbf{Y}$ and $\mathbf{O}$ crystal exhibit phosphorescence.
\end{abstract}

\section{Introduction}

Triphenylmethyl (trityl) cation ${ }^{[1-4]}$ is a classical carbocation that possesses three-fold symmetry and a positive charge that is delocalized over the entire molecule (Figure 1a). Trityl cation, which can be readily prepared by treatment of trityl alcohol with acid, exhibits intense absorption in the visible region. Since the time of the discovery of this cation, organic chemists have conducted extensive studies concentrating on the synthesis and exploration of derivatives including $\pi$-extended analog ${ }^{[5-8]}$ and heteroatom containing ( $\mathrm{N}$ or $\mathrm{O}$ ) systems, as well as on developing methods for stabilizing the cationic state toward reactions with nucleophiles, and tuning its absorption and emission properties. Through these efforts, many highly absorbing and emitting derivatives have been uncovered, including those of malachite green, crystal violet, fluorescein and rhodamine derivatives that are utilized as textile dyes, external stimuli responsiveness fluorescent probes and cell imaging reagents ${ }^{[9-11]}$ (Figure 1b). Furthermore, owing to its high reactivity, the non-substituted (NS) trityl cation has been widely used as a Lewis acid catalyst (Mukaiyama-type aldol or Michael reaction), photooxidation promoter, hydride acceptor and protecting group component. ${ }^{[12-16]}$ In contrast, the optical properties of NS trityl cation have not yet been fully explored likely because of its high reactivity.
Although various new aromatic compounds have been synthesized, knowledge about the fundamental properties of reactive aromatic cations is also useful in the development of cationic dye, sensors and emissive materials. It seems appropriate, that now, 120 years from the time of the first report of the trityl cation, ${ }^{[2,3]}$ we have carried out an investigation exploring the absorption and luminescence features of the NS trityl cation. In this effort, we discovered that in the crystalline state NS trityl cation displays bright luminescence, unique thermochromism, ${ }^{[17-20]}$ and phosphorescence ${ }^{[21-25]}$ which are governed by the arrangement of molecules in the crystal.

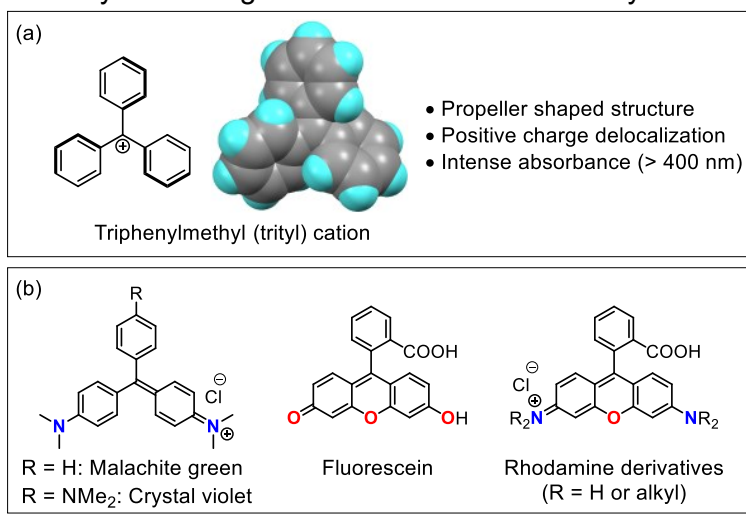

Figure 1. (a) Structure of NS trityl cation. (b) Trityl cation based stable dye fluorescent probe, or emissive imaging materials containing heteroatoms.

\section{Results and Discussion}

\section{Synthesis and crystallization behavior}

The NS trityl cation with $\mathrm{PF}_{6}$ as the counter anion was prepared by treatment of trityl alcohol with hexafluorophosphoric acid $\left(\mathrm{HPF}_{6}\right)$. Specifically, to a solution of trityl alcohol in acetic anhydride at room temperature was added a $60 \% \mathrm{HPF}_{6}$ and the resulting mixture was let stand at room temperature while crystal growth occurred. ${ }^{[26]}$ At first, yellow $(\mathbf{Y})$ crystals formed (Figure $2 a$ b), but when the solution stood for a several ten minute period, production of orange (0) crystal began (Figure 2c, 2d). Finally, upon standing for days to weeks (Figure $2 \mathrm{e}-2 \mathrm{~g}$ ), $\mathrm{O}$-crystal are formed as the major component. The single-crystal-to-singlecrystal (SCSC) phase transition ${ }^{[27-32]}$ from $\mathbf{Y}$ - to O-crystals can be effectively suppressed by keeping the solution at low 
temperature $\left(4{ }^{\circ} \mathrm{C}\right)$. This phenomenon enabled selective isolation of the two crystal polymorphs.

It is known that the colors of trityl salts in the solid state depend on the nature of the counter anion. It was previously reported that the crystal color of trityl $\mathrm{PF}_{6}$ is orange. ${ }^{[33]}$ Furthermore, trityl tetrafluoroborate, prepared to evaluate the counter anion effect on the SCSC transition, was found not to exist as crystal polymorphs but only one yellow crystalline form, which has cell parameters that are similar to those of the with $\mathrm{Y}$ crystal of trityl $\mathrm{PF}_{6}$ (Figure S5). Thus, the nature of the counter anion is crucial for the occurrence of this SCSC phase transition.

The thermal stabilities of the $\mathbf{Y}$ - and $\mathbf{O}$-crystals were elucidated by using differential scanning calorimetry (DSC). The scan of the Y-crystal contains lower endothermic peaks at $104{ }^{\circ} \mathrm{C}$ (phase transition) and $148{ }^{\circ} \mathrm{C}$ (decomposition) and that of O-crystal contains a peak at $168{ }^{\circ} \mathrm{C}$ (decomposition) (Figure S1). In addition, X-ray analysis shows that the calculated crystal density of the 0 -crystal at $273 \mathrm{~K}$ is $1.524 \mathrm{~g} \mathrm{~cm}^{3}$, which is higher than that of the $\mathrm{Y}$-crystal $\left(1.413 \mathrm{~g} \mathrm{~cm}^{3}\right)$. These results indicate that formation of the Y-crystal phase is kinetically preferred and the O-crystal is thermally more stable in a manner that typically exemplifies the Ostwald's step rule. ${ }^{[34-36]}$

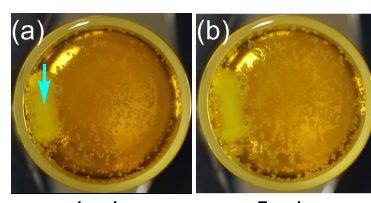

$1 \mathrm{~min}$

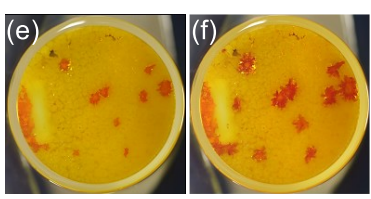

$12 \mathrm{~h}$

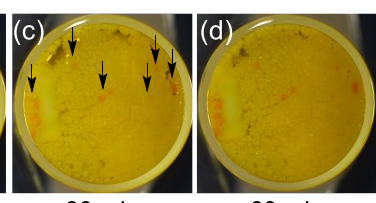

$60 \min$

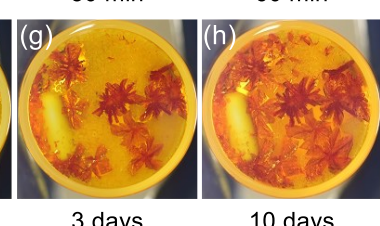

Figure 2. Photographs showing crystal growth and a SCSC phase transition from (a) Y-crystal to (h) O-crystal at elapsed times following initiation of crystallization. The blue arrow in (a) indicates a stir tip as a standard material. The black arrows in (c) indicate the beginning of growth of the O-crystal.

\section{Optical properties and crystal structures}

In Figure $3 a$ are shown the UV-vis spectrum of a $\mathrm{CHCl}_{3}$ solution of the NS trityl cation, generated by reaction of trityl alcohol with $2 \%$ TFA, and solid-state transmission UV-vis spectra of $\mathbf{Y}$ - and $\mathbf{O}$-crystals dispersed in $\mathrm{KBr}$ pellets. The color of the solution of the trityl cation is yellow, corresponding to a $\lambda_{\max }$ at 435 and $410 \mathrm{~nm}\left(\varepsilon=45000 \mathrm{~cm}^{-1}\right.$ $\left.\mathrm{M}^{-1}\right)$. The UV-vis spectrum of the $\mathrm{Y}$-crystal is nearly identical to that of a solution of the trityl cation whereas the spectrum of the O-crystal contains a distinctively bathochromically shifted maximum above $550 \mathrm{~nm}$. This finding was confirmed by inspection of the solid-state diffuse reflectance spectra of these crystals (Figure S2). Although several aromatic cations containing heteroatoms such as $\mathrm{N}$ or $\mathrm{O}$ are known to exhibit intense emission, ${ }^{[37,38]}$ those composed of only hydrogen and carbon exhibit very weak or no emission in solution owing to the favorability of nonradiative decay processes. ${ }^{[39]}$ In fact, the trityl cation in solution displays no emission. On the other hand, both the $\mathbf{Y}$ - and $\mathbf{O}$-crystals of trityl $\mathrm{PF}_{6}$ display relatively intense yellow green $\left(\lambda_{\mathrm{em}}=525 \mathrm{~nm}\right)$ and orange $\left(\lambda_{\mathrm{em}}=585 \mathrm{~nm}\right)$ emission, respectively (Figure $3 b$ ). The emission quantum yields of the $\mathbf{Y}$ - and $\mathbf{O}$-crystals at $293 \mathrm{~K}$ are $4 \%$ and $12 \%$, respectively. This phenomenon is a consequence of aggregation induced emission (AIE) ${ }^{[40-48]}$ because radiationless decay associated with rotation of the three phenyl rings is effectively suppressed in the crystalline state. To the best of our knowledge, no hydrocarbon cations and

only a few aromatic cations containing $\mathrm{N}$ and $\mathrm{O}$ atoms have been reported to exhibit AIE. ${ }^{[23,48]}$

To gain information about the optical differences between the $\mathbf{Y}$ - and $\mathbf{O}$-crystals, single crystal $\mathbf{X}$-ray analysis was performed to determine crystal packing structures. The results show that the trityl cation in the Y-crystal at $170 \mathrm{~K}$ has $D_{3}$ symmetry with a torsion angle of the three phenyl rings being $32.9^{\circ}$ (Figure S3). In the unit structure of the $\mathbf{Y}$ crystal, four trityl cations are oriented in a tetrahedral arrangement (Figure $4 \mathrm{a}, \mathrm{b}$ ) and the $\mathrm{PF}_{6}$ anions are located in a central cavity as well as in areas surrounding the tetrahedron (Figure 4c). Thus, each trityl cation in the $\mathbf{Y}$ crystal is completely isolated from neighboring cations by intervening $\mathrm{PF}_{6}$ anions and, as a result, no significant interactions exist between the trityl scaffolds. As stated above, the molecular arrangement in the Y-crystal is reflected in its UV-vis spectrum, which is similar to that of the solution state.
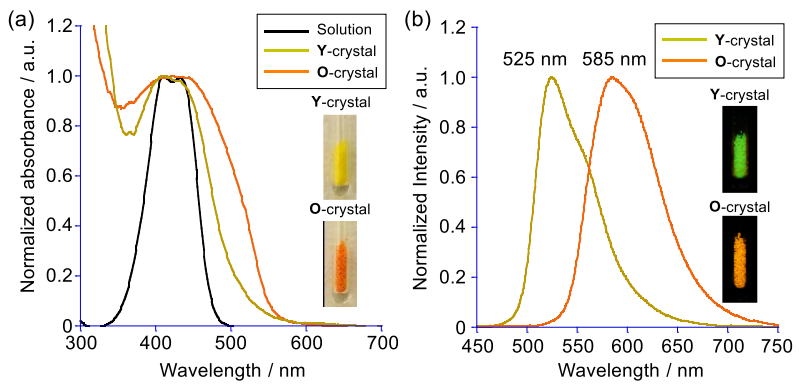

Figure 3. (a) UV-vis spectrum of trityl cation (2\% TFA in $\left.\mathrm{CHCl}_{3}\right)$ and solidstate UV-vis spectra of $\mathbf{Y}$ - and $\mathbf{O}$-crystals ( $\mathrm{KBr}$ pellet). (b) Emission spectra of Y- and O-crystals $\left(\lambda_{\mathrm{ex}}=360 \mathrm{~nm}\right)$

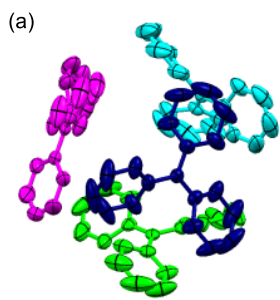

(b)

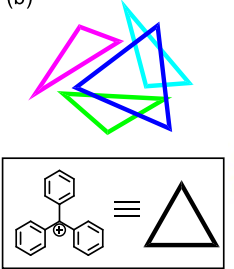

(c)

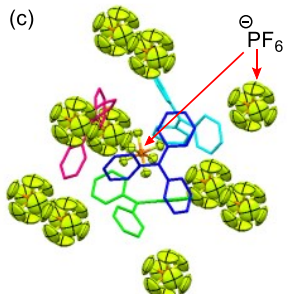

Figure 4. X-ray crystallographic analysis of Y-crystal (170 K). (a) Tetrahedral arrangement of trityl cations depicting different colors without $\mathrm{PF}_{6}$ anions for clarity. (b) Schematic drawing of the tetrahedral arrangement of trityl cations as shown in (a). (c) Drawing with $\mathrm{PF}_{6}$ anions shown in (a). Protons are omitted for clarity. Ortep drawing is $50 \%$ probability.

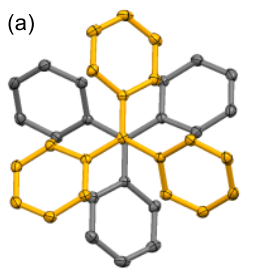

(c)

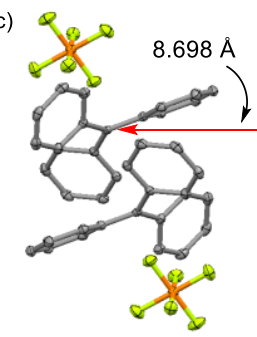

Figure 5. X-ray crystallographic analysis of O-crystal (195 K). (a) Top view of trityl cation dimer in O-crystal with different colors and without $\mathrm{PF}_{6}$ anions for clarity. (b) Side view of trityl $\mathrm{PF}_{6}$ dimer in O-crystal with $\mathrm{PF}_{6}$ anions. (c) One dimensional alignment of trityl $\mathrm{PF}_{6}$ (viewing from $c$ axis). Protons are omitted for clarity. Ortep drawing is $50 \%$ probability. 
In a manner that is different from that of the in the $Y$ crystal, the trityl cation scaffold in the $\mathbf{O}$-crystal has $C_{1}$ symmetry with the different torsion angles between the three phenyl rings being $25.4,31.8$ and $40.2^{\circ}$ (Table S2). Two types of aggregation arrangements exist in the packing structure of the $\mathbf{0}$-crystal. In one of these, two trityl cations form a face-to-face dimer with $\mathrm{PF}_{6}$ anions at exterior positions (Figure 5a, b). The intermolecular atomic distance between the central $\mathrm{sp}^{2}$ carbon of the trityl cations is 4.265 $\AA(195 \mathrm{~K})$, which is ca. $1.0 \AA$ greater than the distance for typical $\pi-\pi$ stacking as a result of steric hindrance between the phenyl rings and positive charge repulsion. In the other arrangement, the trityl cations are one dimensionally laterally aligned with a periodic distance of $8.698 \AA$ (Figure $5 \mathrm{c})$. It is noteworthy that the inter-atomic $\mathrm{C} \cdots \mathrm{F}$ distance between central $\mathrm{sp}^{2}$ carbon of the trityl cation and fluorine atom in $\mathrm{PF}_{6}$ anion is $3.101 \AA(195 \mathrm{~K})$ (Figure $5 \mathrm{~b}$ ), a value that is almost identical to the sum of the van der Waals $(v d W s)$ radii of carbon $(1.70 \AA)$ and fluorine $(1.47 \AA)$ atoms. This observation indicates the existence of a stronger anion- $\pi^{+}$interaction ${ }^{[49-52]}$ than that existing in the $\mathbf{Y}$-crystal (C $\cdots \mathrm{F}$ distance is $3.503 \AA$ at $170 \mathrm{~K}$ ). In fact, the results of natural bond orbital (NBO) analysis of trityl $\mathrm{PF}_{6}$ monomer in both $\mathrm{Y}$ - and $\mathbf{O}$-crystals using MP2/6-311+G** reveals that a charge-transfer (CT) interaction is present between the trityl cation and $\mathrm{PF}_{6}$ anion ${ }^{[49]}$ and that the degree of the CT interaction in the $\mathbf{O}$-crystal is larger than it is in the $\mathbf{Y}$-crystal (Figure S8). In addition, the NBO analysis suggests that the face-to-face trityl $\mathrm{PF}_{6}$ dimeric structure in $\mathbf{O}$-crystal enables a greater degree of $\mathrm{CT}$ interaction than that which exists in the trityl $\mathrm{PF}_{6}$ monomer (Figure S9). This is likely caused by the unfavorable cation-cation repulsive energy in the faceto-face dimeric structure which enhances $\mathrm{CT}$ interaction from the $\mathrm{PF}_{6}$ anion to the trityl cation.

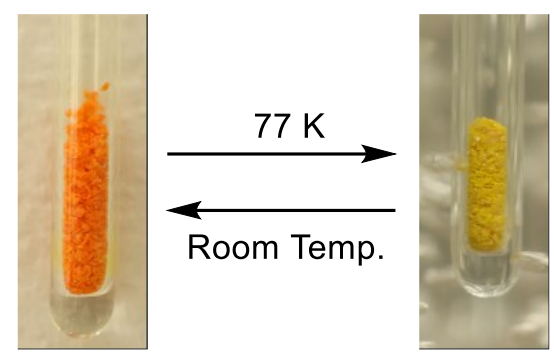

Figure 6. Thermochromism of the 0-crystal between room temperature and $77 \mathrm{~K}$. The detail of this thermochromism can be see in Movie S1 (under room light) and Movie S2 (under UV light).
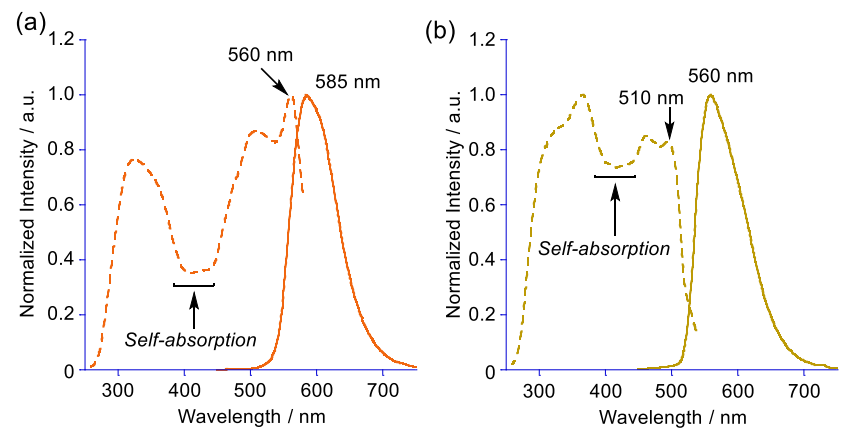

Figure 7. (a) Excitation (dash line) and emission (solid line) spectra of $\mathbf{O}$ crystal at $293 \mathrm{~K}$. (b) Excitation (dash line) and emission (solid line) spectra of O-crystal at $77 \mathrm{~K}$. In both excitation spectra, self-absorption from 400 to 450 $\mathrm{nm}$ is due to the intense absorption on the crystal surface. ( $\lambda_{\mathrm{ex}}=360 \mathrm{~nm}$ for emission measurements, $\lambda_{\mathrm{em}}=585 \mathrm{~nm}$ in $293 \mathrm{~K}$ and $\lambda_{\mathrm{em}}=560 \mathrm{~nm}$ in 77 $\mathrm{K}$ for excitation measurements.)

\section{Thermochromism of O-crystal}

Additional investigations led to the discovery of other interesting properties of the O-crystal of trityl $\mathrm{PF}_{6}$. Upon cooling the temperature from room temperature to $77 \mathrm{~K}$ the color of $\mathbf{O}$-crystal changed to yellow and the orange color reformed upon warming to room temperature (Figure 6 and Movie S1). Similar to these absorption changes, the emission color of $\mathbf{O}$-crystal changes from orange to yellow orange upon cooling (Movie S2). This type of distinctive thermochromism does not take place with the Y-crystal (Movie S3-4, Figure S10). The thermochromic behavior of the O-crystal was evaluated by using emission and excitation spectroscopic measurements (Figure 7a, b). The excitation spectrum at room temperature contains a peak at $560 \mathrm{~nm}$ whereas at $77 \mathrm{~K}$ the maximum occurs at a wavelength shorter than $560 \mathrm{~nm}$. In addition, the emission peak also shifts from $585 \mathrm{~nm}$ to $560 \mathrm{~nm}$ when the temperature is lowered from room temperature to $77 \mathrm{~K}$.

To evaluate the relationship between thermochromism and structural changes, single crystal $X$ ray analysis was conducted on the 0 -crystal at 273 and 83 $\mathrm{K}$ (Figure S5, S6). The results show that upon cooling, no change occurs in the crystal system (monoclinic, $P 2_{1} / n$ ) and negligible changes take place in the torsion angles of three phenyl rings, as well as in $\mathrm{C}-\mathrm{C}$ bond lengths between the central $\mathrm{sp}^{2}$ carbon and ipso-phenyl carbons (Table S2). On the other hand, the inter-atomic $\mathrm{C} \cdots \mathrm{F}$ distance between fluorine atom of the $\mathrm{PF}_{6}$ anion and the central $\mathrm{sp}^{2}$ carbon of trityl cation upon cooling shortens from $3.155 \AA$ (273 K) to $3.047 \AA(83 \mathrm{~K})$ (Figure $8 \mathrm{a}$ and Figure S5, S6), which is shorter than the sum of vdWs radii of carbon and fluorine atoms. In addition, the distance between the central $\mathrm{sp}^{2}$ carbons in face-to-face trityl cation dimers in the crystal are slightly reduced from $4.296 \AA(273 \mathrm{~K})$ to $4.232 \AA(83 \mathrm{~K})$ (Figure $8 \mathrm{~b}$ ) upon cooling. The cause of this change is reduction of the net charge on the trityl cation by strong $C T$ from the $\mathrm{PF}_{6}$ anion, which is confirmed by using Hirshfeld atomic population analysis (MP2/6-311+G**, Table S3).

TD-DFT calculations of trityl $\mathrm{PF}_{6}$ in the O-crystal at both 273 and $83 \mathrm{~K}$ were carried out using CAM-B3LYP functional with a tuned parameter method $(\mu=0.150, \alpha=$ 0.0799, $\beta=0.9201(\alpha+\beta=1.0))$ as developed by Nakano. ${ }^{[53]}$ The results reveal that the longer wavelength transitions $(430.4,429.6$ and $424.8 \mathrm{~nm}$ with respective $f=$ $0.0004,0.0035$ and 0.0116 , Table S4 and Figure S11, S13) of the crystal at $273 \mathrm{~K}$, containing two trityl cations arranged in a face-to-face dimer orientation, are associated with a weak $\mathrm{CT}$ transition from the $\mathrm{PF}_{6}$ anion to the trityl cation. The major band at $83 \mathrm{~K}$ corresponds to an intramolecular transition in trityl cation $(422.0 \mathrm{~nm}, f=0.0441)$ because shortening of the $\mathrm{C} \cdots \mathrm{F}$ distance leads to strengthen of the CT transition and shifting it to higher energy (406.1, 404.3 and $402.7 \mathrm{~nm}$ with respective $f=0.0052,0.0039$ and 0.0438 , Table S5 and Figure S12). In contrast, calculations on crystals containing a trityl $\mathrm{PF}_{6}$ monomer and a laterally arranged trityl $\mathrm{PF}_{6}$ dimer, show that little change occurs in the longer wavelength transition upon changing the temperature between 273 and $83 \mathrm{~K}$ (Table S6-S8 and Figure S14-20). Therefore, the face-to-face dimer structure promoted perturbation of the CT transition energy through a cation-cation repulsive interaction is the cause of the prominent color change from orange to yellow that occurs upon cooling the O-crystal. 
(a)

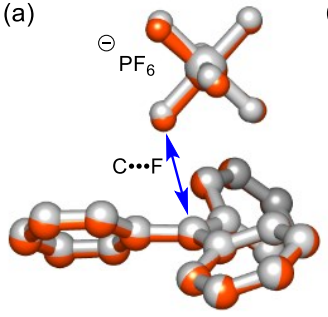

C...F distance

$273 \mathrm{~K}: 3.155 \AA$

$83 \mathrm{~K}: 3.047 \AA$ (b)

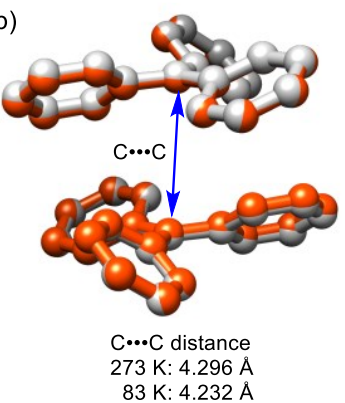

Figure 8. Superposition of the structure generated by VT X-ray analysis of $\mathbf{O}$ crystal at $273 \mathrm{~K}$ (grey) and $83 \mathrm{~K}$ (orange). (a) Depicting the structural changes between $\mathrm{PF}_{6}$ anion and trityl cation. (b) Depicting the structural changes between face-to-face trityl cation dimer.

\section{Phosphorescence in trityl cation}

The trityl cation is isoelectronic with triphenylborane, which displays unique optical properties including thermally activated delayed fluorescence (TADF). ${ }^{[54-59]}$ Owing to this relationship, we explored the delayed emissive nature of a solid solution of trityl $\mathrm{PF}_{6}$. The emission spectrum of a solid solution of trityl cation $\left(5.0 \times 10^{-6} \mathrm{M}\right)$ in $\mathrm{CHCl}_{3}$ with $2 \%$ TFA at $77 \mathrm{~K}$ contains a broad weak peak from 510 to $520 \mathrm{~nm}$ and an intense peak at $550 \mathrm{~nm}$ (Figure 9a). Delayed emission measurements (10 ms delay) show that the broad and weak $510-520 \mathrm{~nm}$ peak disappears and an intense emission band at $550 \mathrm{~nm}$ arises. It is noteworthy that the same sample at $195 \mathrm{~K}$ contains a broad delayed emission band $515 \mathrm{~nm}$ (Figure S21). The broad 510-520 nm and intense $550 \mathrm{~nm}$ peaks are assigned as respective fluorescence and phosphorescence of trityl cation. Thus, a remarkably small energy gap $\left(\Delta E_{S T}\right)$ of $0.15 \mathrm{eV}$ exists between $S_{1}$ and $T_{1}$ of this substance. The lifetime of the phosphorescence of trityl cation is $310 \mathrm{~ms}$ (Figure S23) and the emission quantum yield is $40 \%$ (Table 1).

Table 1. Emission peaks, lifetime, and quantum yield of trityl cation (77 K), Y-crystal (293 and $77 \mathrm{~K})$, and O-crystal (293 and $77 \mathrm{~K})$.

\begin{tabular}{llll}
\hline & $\lambda_{\mathrm{em}} / \mathrm{nm}$ & T & $\Phi / \%[\mathrm{~d}]$ \\
\hline $\begin{array}{l}\text { Trityl } \\
\text { cation } \\
(77 \mathrm{~K})^{[\mathrm{a}]}\end{array}$ & 510,550 & $310 \mathrm{~ms}(550 \mathrm{~nm})$ & 40 \\
\hline Y-cryst. & 525 & $1.8 \mathrm{~ns}(525 \mathrm{~nm})$ & 4 \\
$(293 \mathrm{~K})$ & & & \\
\hline Y-cryst. & $510(\mathrm{SS})^{[\mathrm{b}]}$ & $340 \mathrm{~ms}(550 \mathrm{~nm})$ & 11 \\
$(77 \mathrm{~K})$ & $550,585(\mathrm{P})^{[\mathrm{c}]}$ & & 12 \\
\hline $\begin{array}{l}\text { O-cryst. } \\
(293 \mathrm{~K})\end{array}$ & 585 & $9.1 \mathrm{~ns}(575 \mathrm{~nm})$ & \\
\hline O-cryst. & $560(\mathrm{SS})^{[\mathrm{b}]}$ & & \\
$(77 \mathrm{~K})$ & $550,617(\mathrm{P})^{[\mathrm{c}]}$ & $410 \mathrm{~ms}(560 \mathrm{~nm})$ & 24 \\
\hline
\end{tabular}

[a] Trityl cation was generated from trityl alcohol in $\mathrm{CHCl}_{3}$ with $2 \%$ TFA. [b] SS indicates steady-state emission. [c] $P$ indicates phosphorescence emission. [d] Emission quantum yield contains both fluorescence and phosphorescence measured by using integrating sphere.

In contrast to those of the frozen state, the phosphorescence emission properties of the $\mathbf{Y}$ - and $\mathbf{O}$-crystals are totally different. The steady-state (SS) emission spectrum of Y-crystal at $77 \mathrm{~K}$ contains an intense peak at $510 \mathrm{~nm}$, which is nearly the same wavelength as that in the fluorescence spectrum of frozen state of trityl cation. The phosphorescence emission spectrum of the $\mathbf{Y}$-crystal contains two peaks at 550 and $585 \mathrm{~nm}$ (Figure 9b) associated with an emission lifetime of $340 \mathrm{~ms}$, which is comparable with that of the frozen state. On the other hand, the SS emission spectral band of the O-crystal begins at $480 \mathrm{~nm}$ with a maximum at $560 \mathrm{~nm}$. In addition, the SS emission spectrum overlaps with that of its phosphorescence spectrum (Figure 9c). ${ }^{[60]}$ Although the trityl cation has a small $\Delta E_{\mathrm{ST}}$, the emission quantum yield of the O-crystal is enhanced by cooling $(12 \%$ at $293 \mathrm{~K}$ to $24 \%$ at $77 \mathrm{~K}$ ), which rules out the existence of TADF. Therefore, emission occurs from the triplet state as phosphorescence, as shown in the SS emission spectrum of the $\mathbf{0}$-crystal at $77 \mathrm{~K}$.

The emission lifetime of the O-crystal at $293 \mathrm{~K}$ is $9.1 \mathrm{~ns}$ (Figure S24) whereas at $77 \mathrm{~K}$ it is $410 \mathrm{~ms}$, which is slightly longer than those of the frozen solution state and the $\mathbf{Y}$-crystal. Longer wavelength emission peaks above $550 \mathrm{~nm}$ are present in the phosphorescence emission spectra of the $\mathbf{Y}$-crystal $\left(\lambda_{\mathrm{em}}=\right.$ $585 \mathrm{~nm})$ and O-crystal $\left(\lambda_{\mathrm{em}}=617 \mathrm{~nm}\right)$. Phosphorescence spectra caused by excitation at 550 and $590 \mathrm{~nm}$ (for Y-crystal) and $620 \mathrm{~nm}$ (for O-crystal), were analyzed. Interestingly, a spectra that has a shape that is similar to that of the an absorption spectrum of trityl cation when excitation occurs at at $550 \mathrm{~nm}$ whereas sharp longer wavelength shifted excitation spectra of both the $\mathbf{Y}$ - and $\mathbf{O}$-crystal are promoted by using 590 and $620 \mathrm{~nm}$ excitation, respectively (Figure 10). Furthermore excitation at $480 \mathrm{~nm}$ for Y-crystal and $500 \mathrm{~nm}$ for O-crystal leads to selected enhancements of the emission peaks at $580 \sim 585 \mathrm{~nm}$ for Y-crystal and $620 \mathrm{~nm}$ for O-crystal. On the other hand, the emission spectrum of the frozen state displays no dependence on excitation wavelength and concentration (Figure S22). Therefore, these longer wavelength phosphorescence peaks are attributed from their packing structures such as the arrangement of trityl cations whereas the emission peak at $550 \mathrm{~nm}$ is attributed from the monomer structure of trityl cation. Inspection of the ns to $\mu$ s timescale emission decay profiles of the $\mathbf{Y}$ - and O-crystals at $77 \mathrm{~K}$ shows that, in contrast to the frozen state, both $\mathbf{Y}$ - and $\mathbf{O}$-crystal display intense emission in nanosecond region (<240 ns) and relatively weak emission in microsecond region (Figure S25). Owing to the weakness of emission in the microsecond region, analysis of the long time decay up to the millisecond timescale was difficult to perform using our instrumental setup and, thus, the phosphorescence quantum yield could not be determined.

Spin-orbit coupling (SOC) constants were calculated using the TD-DFT method with Tamm-Dancoff approximation (TDA) to evaluate the phosphorescence properties of the trityl cation. It is noteworthy that small SOCs occur between the $S_{0}$ and $T_{1}$ states as well as $S_{1}$ and $T_{n}$ states of the trityl cation alone (Figure S26) whereas significant SOCs takes place between singlet and triplet states in the ion pair of trityl cation and $\mathrm{PF}_{6}$ anion (Figure S27-S29). This finding indicates that ISC of the trityl cation is facilitated by the presence of the counter anion. Judging from the energies of the singlet and triplet excited states of trityl $\mathrm{PF}_{6}$ ion pair in the O-crystal at $273 \mathrm{~K}$, the energies of $\mathrm{S}_{1,2}$, which are almost degenerated energy states, are close to those of $\mathrm{T}_{6-8}$ and a large SOC exists between these states, resulting in effective ISC and enabling subsequent internal conversion $T_{6-8}->T_{1}$.

The calculations also show that, although only small differences exist in the degree of SOC between trityl $\mathrm{PF}_{6}$ ion pairs in the $\mathbf{Y}$ - and $\mathbf{O}$-crystals as well as in the $\mathbf{O}$-crystal between 273 and $83 \mathrm{~K}$, a distinctive enhancement of the SOC exists in the face-to-face dimer of trityl $\mathrm{PF}_{6}$ ion pair structure in the 
(a)

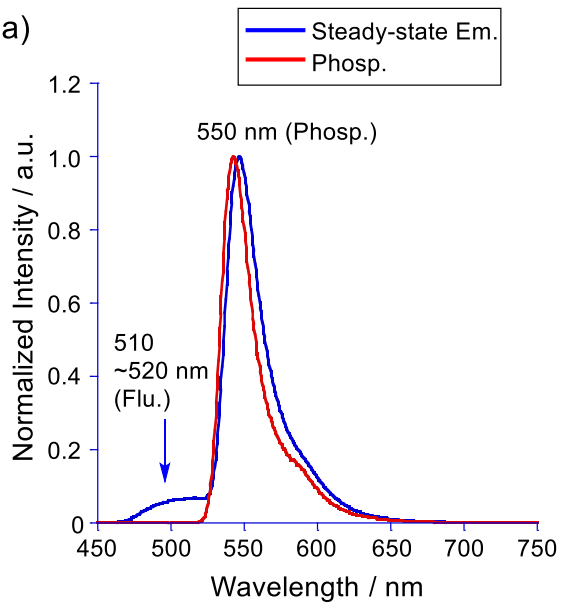

(b)

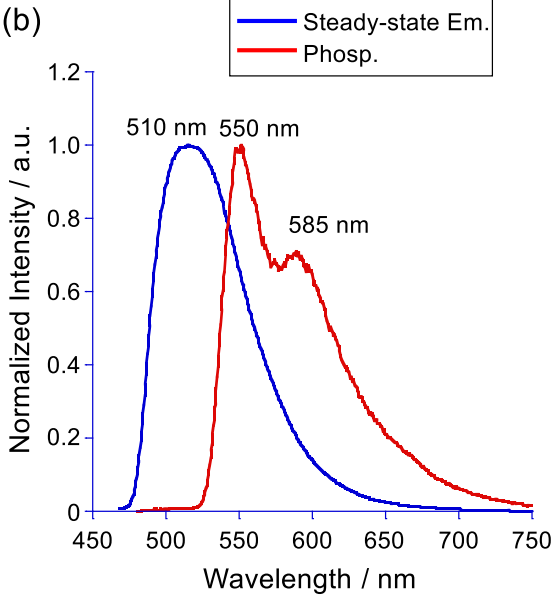

(c)

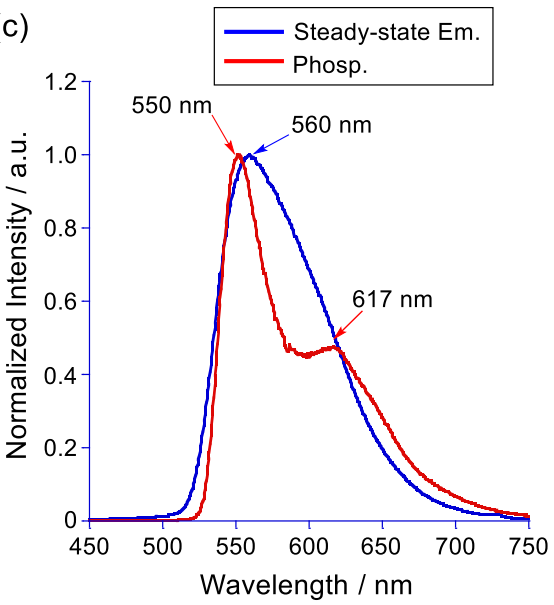

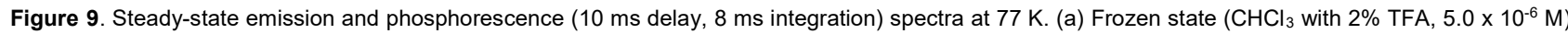
of trityl cation. (b) Y-crystal. (c) O-crystal. $\left(\lambda_{\mathrm{ex}}=360 \mathrm{~nm}\right)$

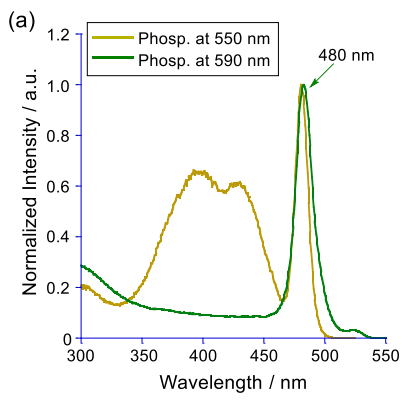

(b)
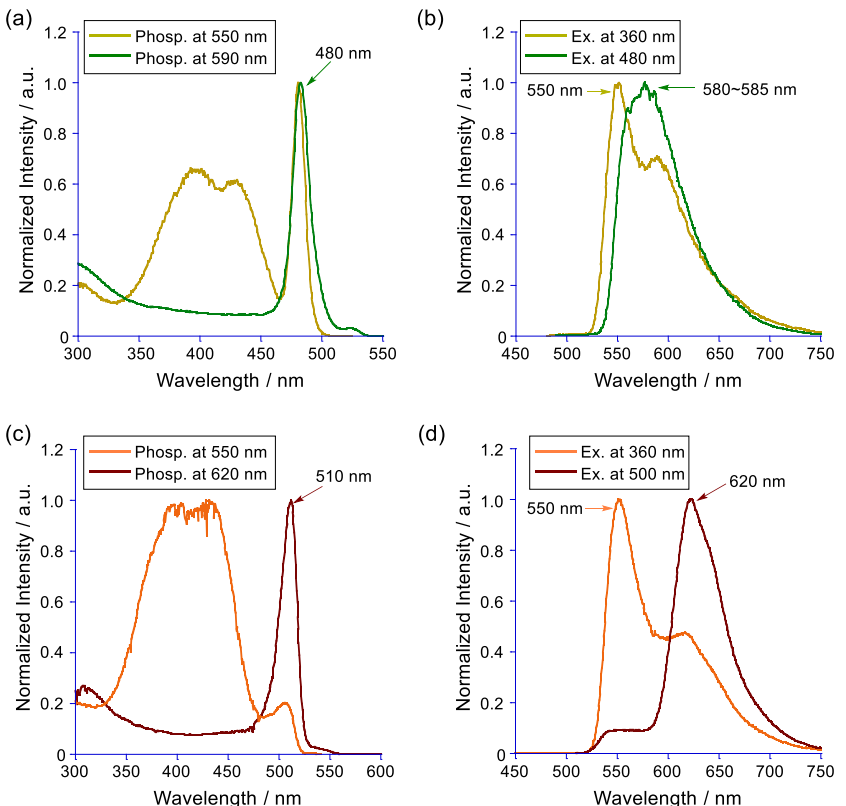

(d)

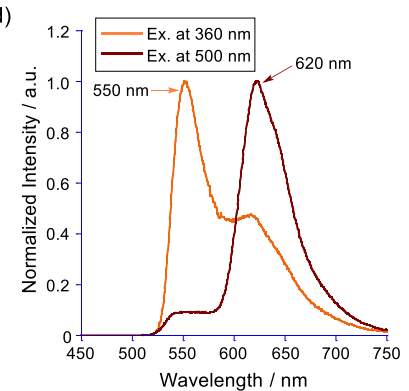

Figure 10. Phosphorescence excitation spectra and excitation wavelength dependence of phosphorescence of Y-crystal (a),(b) and O-crystal (c),(d). Spectra were measured with a $10 \mathrm{~ms}$ delay and $8 \mathrm{~ms}$ integration at $77 \mathrm{~K}$.

O-crystal. For example, the $\mathrm{S}_{0} / \mathrm{T}_{1} \mathrm{SOC}$ value at $273 \mathrm{~K}$ for the monomer is $2.54 \mathrm{~cm}^{-1}$ (monomer) whereas it is $51.1 \mathrm{~cm}^{-1}$ for the face-to-face dimer. In addition, ISC of the dimer is greater than that of the monomer (Figure S30). Moreover, the SOC values also depend on the distance between trityl cation and $\mathrm{PF}_{6}$ anion, as reflected by the the increase in the $S_{0} / T_{1} S O C$ value to 80.2 $\mathrm{cm}^{-1}$ at $83 \mathrm{~K}$ (Figure S31). These results indicate that the faceto-face cation dimer structural arrangement has a drastic effect on photophysical properties and that the temperature tunable distance between the cation and anion enables control of the emission properties.

\section{Conclusion}

In the investigation described above, we uncovered the unique optical nature of the non-substituted trityl cation, which is a fundamental aromatic hydrocarbon cation. The studies show that crystalline trityl $\mathrm{PF}_{6}$ exhibits a SCSC phase transition from Y- to O-crystals, which originates from $\mathrm{CT}$ interactions between the trityl cation and $\mathrm{PF}_{6}$ anion. In addition, trityl $\mathrm{PF}_{6}$ displays crystalline state fluorescence and phosphorescence. Importantly, the molecular arrangement of the trityl cation and $\mathrm{PF}_{6}$ anion in the crystalline state plays a dominant role in governing photophysical properties. This phenomenon was explored by using TD-DFT as well as SOC calculations, which gave a more detailed understanding of how ion arrangements in the Y-and O-crystals govern excited properties. We believe that other hydrocarbon cations have the potential of possessing these unique optical properties. It is anticipated that the results of the current effort will pave the way to an exploration of hydrocarbon cations and their use in organic luminescent devices, and in switching and sensing systems.

\section{Acknowledgements}

Triphenylmethanol used for the synthesis of the trityl cation was provided by an experimental class of bachelor students in Department of Chemistry, Osaka University. T. N. would like to thank Prof. Takumi Konno and Prof. Nobuto Yoshinari (Osaka Univ.) for helping to measure emission and excitation spectra, Prof. Shuichi Suzuki (Osaka Univ.) for helping to measure DSC and Prof. Yasutaka Kitagawa (Osaka Univ.) for fruitful discussions to calculate and evaluate the SOCs of trityl cations. The single crystal X-ray analyses were performed at the Analytical Instrument Facility, Graduate School of Science, Osaka University. The computations were performed at the Research Center for Computational Science, Okazaki, Japan. This study was supported by JSPS KAKENHI Grant-in-Aid for Scientific Research (C), Grant Number JP20K05475 (T.N.) and for Scientific Research (B), Grant Number JP21H01887 (K.K.).

\section{Conflict of interest}

The authors declare no conflict of interest. 
Keywords: carbocation • aggregation induced emission • single crystal-to-single crystal transition • thermochromism• phosphorescence

[1] Reviews: "Arylcarbonium lons": H. H. Freedman, Carbonium lons, Vol IV (Eds.: G. A. Olah, P. von R. Schleyer), Wiley-Interscience, New York 1972, pp. 1501.

[2] J. F. Norris, Am. Chem. J. 1901, 25, 117.

[3] F. Kehrmann, F. Wentzel, Ber. Dtsch.Chem. Ges. 1901, 34, 3815

[4] A. Baeyer, V. Villiger, Ber. Dtsch.Chem. Ges. 1902, 35, 1189.

[5] G. A. Olah, Q. Liao, J. Casanova, R. Bau, G. Rasul, G. K. S. Prakash, J. Chem. Soc. Perkin Trans. 2. 1998, 2239.

[6] S. Ito, N. Morita, T. Asao, Bull. Chem. Soc. Jpn. 1995, 68, 1409

[7] Y. Nishimae, H. Kurata, M. Oda, Angew. Chem. Int. Ed. 2004, 43, 4947.

[8] T. Nishiuchi, S. Aibara, T. Kubo, Angew. Chem. Int. Ed. 2018, 57, 16516.

[9] D. F. Duxbury, Chem. Rev. 1993, 93, 381

[10] V. Nair, S. Thomas, S. C. Mathew, K. G. Abhilash, Tetrahedron. 2006, 62, 6731.

[11] T. Gessner, U. Mayer, "Triarylmethane and Diarylmethane Dyes" Ullmann's Encyclopedia of Industrial Chemistry, Wiley- $\mathrm{VCH}$, Weinheim 2000

[12] S. Kobayashi, M. Murakami, T. Mukaiyama, Chem. Lett. 1985, 14, 1535.

[13] T. Mukaiyama, M. Tamura, S. Kobayashi, Chem. Lett. 1986, 15, 1017.

[14] C. J. Urch, "Triphenylmethyl Hexafluorophosphate" In Encyclopedia of Reagents for Organic Synthesis, 2001.

[15] M. E. Jung, R. Lagoutte, U. Jahn, "Triphenylcarbenium Tetrafluoroborate" In Encyclopedia of Reagents for Organic Synthesis, 2011.

[16] M. S. Shchepinov, V. A. Korshun, Chem. Soc. Rev. 2003, 32, 170.

[17] R. R. Chance, R. H. Baughman, H. Müller, C. J. Eckhardt, J. Chem. Phys. 1977, 67, 3616.

[18] E. Hadjoudis, I. M. Mavridis, Chem. Soc. Rev. 2004, 33, 579

[19] Y. Morita, S. Suzuki, K. Fukui, S. Nakazawa, H. Kitagawa, H. Kishida H. Okamoto, A Naito, A Sekine, Y Ohashi, M. Shiro, K. Sasaki, D. Shiomi, K. Sato, T. Takui, K. Nakasuji, Nat. Mater. 2008, 7, 48.

[20] T. Kinuta, T. Sato, N. Tajima, R. Kuroda, Y. Matsubara, Y. Imai, J. Mol. Struct. 2010, 982, 45.

[21] X. Sun, B. Zhang, X. Li, C. O. Trindle, G. Zhang, J. Phys. Chem. A 2016, 120, 5791 .

[22] S. Hirata, Adv. Opt. Mater. 2017, 5, 1700116.

[23] J. Wang, X. Gu, H. Ma, Q. Peng, X. Huang, X. Zheng, S. H. P. Sung, G. Shan, J. W. Y. Lam, Z. Shuai, B. Z. Tang, Nat. Commun, 2018, 9, 2963.

[24] K. C. Chen, B. Liu, Nat. Commun. 2019, 10, 2111.

[25] Y. Tani, M. Terasaki, M. Komura, T. Ogawa, J. Mater. Chem. C. 2019, 7, 11926.

[26] G. A. Olah, J. J. Svoboda, J. A. Olah, Synthesis. 1972, 544.

[27] J. Bernstein, "Polymorphism in Molecular Crystals" Oxford Univ. Press 2002.

[28] T. Mutai, H. Sato, K. Araki, Nat. Mater. 2005, 4, 685

[29] S. Kobatake, S. Takami, H. Muto, T. Ishikawa, M. Irie, Nature. 2007, 446, 778.

[30] I. Halasz, Cryst. Growth Des. 2010, 10, 2817.

[31] H. Ito, M. Muromoto, S. Kurenuma, S. Ishizuka, N. Kitamura, H. Sato, T. Seki, Nat. Commun. 2013, 4, 2009.

[32] T. Seki, K. Sakurada, H. Ito, Angew. Chem. Int. Ed. 2013, 52, 12828.

[33] A. Hinz, R. Labbow, F. Reiss, A. Schulz, K. Sievert, A. Villinger, Struct. Chem. 2015, 26, 1641

[34] W. Ostwald, Z. Phys. Chem. 1879, 22, 289.

[35] R. A. Van Santen, J. Phys. Chem. 1984, 88, 5768

[36] T. Threlfall, Org. Proc. Res. Dev. 2003, 7, 1017.

37] K. Asai, A. Fukazawa, S. Yamaguchi, Chem. Eur. J. 2016, 22, 17571.

[38] K. Asai, A. Fukazawa, S. Yamaguchi, Angew. Chem. Int. Ed. 2017, 56 6848.

[39] A. Samanta, K. R. Gopidas, P. K. Das, J. Phys. Chem. 1993, 97, 1583.

[40] R. Deans, J. Kim, M. R. Machacek, T. M. Swager, J. Am. Chem. Soc 2000, 122, 8565 .

[41] J. Luo, Z. Xie, J. W. Y. Lam, L. Cheng, H. Chen, C. Qiu, H. S. Kwok, X Zhan, Y. Liu, D. Zhu, B. Z. Tang, Chem. Commun. 2001, 1740

[42] B. K. An, S. D. Kwon, S. D. Jung, S. Y. Park, J. Am. Chem. Soc. 2002 124,14410

[43] M. Shimizu, Y. Takeda, M. Higashi, T. Hiyama, Angew. Chem. Int. Ed. 2009, 48, 3653.

[44] M. Shimizu, T. Hiyama, Chem. Asian. J. 2010, 5, 1516.

[45] T. Nishiuchi, K. Tanaka, Y. Kuwatani, J. Sung, T. Nishinaga, D. Kim, M. lyoda, Chem. Eur. J. 2013, 19, 4110.

[46] T. Nishiuchi, M. Iyoda, Bull. Chem. Soc. Jpn. 2014, 87, 960

[47] J. Mei, N. L. C. Leung, R. T. K. Kwok, J. W. Y. Lam, B. Z. Tang, Chem Rev. 2015, 115, 11718

[48] J. Wang, X. Gu, P. Zhang, X. Huang, X. Zheng, M. Chen, H. Feng, R. T. K. Kwok, J. W. Y. Lam, B. Z. Tang, J. Am. Chem. Soc. 2017, 139, 16974.

[49] Y. S. Rosokha, S. V. Lindeman, S. V. Rosokha, J. K. Kochi, Angew. Chem. Int. Ed. 2004, 43, 4650.
[50] B. L. Schottel, H. T. Chifotides, K. R. Dunbar, Chem. Soc. Rev. 2008 37, 68.

[51] A. Frontera, P. Gamez, M. Mascal, T. J. Mooibroek, J. Reedijk, Angew. Chem. Int. Ed. 2011, 50, 9564.

[52] M. Giese, M. Albrecht, K. Rissanen, Chem. Rev. 2015, 115, 8867.

[53] K. Okuno, Y. Shigeta, R. Kishi, M. Nakano, Chem. Phys. Lett. 2013, 585, 201.

[54] C. A. Parker, C. G. Hatchard, Trans. Faraday Soc. 1961, 57, 1894

[55] A. Maciejewski, M. Szymanski, R. P. Steer, J. Phys. Chem. 1986, 90 6314.

[56] A. Endo, K. Sato, K. Yoshimura, T. Kai, A. Kawada, H. Miyazaki, C. Adachi, Appl. Phys. Lett. 2011, 98, 083302.

[57] H. Uoyama, K. Goushi, K. Shizu, H. Nomura, C. Adachi, Nature. 2012, 492, 234.

[58] T. Hatakeyama, K. Shiren, K. Nakajima, S. Nomura, S. Nakatsuka, K Kinoshita, J. Ni, Y. Ono, T. Ikuta, Adv. Mater. 2016, 28, 2777.

[59] Z. Yang, Z. Mao, Z. Xie, Y. Zhang, S. Liu, J. Zhao, J. Xu, Z. Chi, M. P. Aldred, Chem. Soc. Rev. 2017, 46, 915.

[60] The reason for the absence of an emission peak $\lambda_{\text {em }}=510 \mathrm{~nm}$ originating from the trityl cation monomer in O-crystal at $77 \mathrm{~K}$ is the occurrence of self-absorption occurs, which is confirmed by viewing the excitation spectra shown in Figure $7 \mathrm{~b}$ and Figure 10c. 